\title{
Design of asynchronous phase detection algorithms optimized for wide frequency response
}

\author{
Daniel Crespo, Juan Antonio Quiroga, and Jose Antonio Gomez-Pedrero
}

\begin{abstract}
In many fringe pattern processing applications the local phase has to be obtained from a sinusoidal irradiance signal with unknown local frequency. This process is called asynchronous phase demodulation. Existing algorithms for asynchronous phase detection, or asynchronous algorithms, have been designed to yield no algebraic error in the recovered value of the phase for any signal frequency. However, each asynchronous algorithm has a characteristic frequency response curve. Existing asynchronous algorithms present a range of frequencies with low response, reaching zero for particular values of the signal frequency. For real noisy signals, low response implies a low signal-to-noise ratio in the recovered phase and therefore unreliable results. We present a new Fourier-based methodology for designing asynchronous algorithms with any user-defined frequency response curve and known limit of algebraic error. We show how asynchronous algorithms designed with this method can have better properties for real conditions of noise and signal frequency variation. (c) 2006 Optical Society of America

OCIS codes: $100.0100,100.2650,100.6890,120.5050$.
\end{abstract}

\section{Introduction}

Many optical methods of measurement deliver the information as a fringe pattern. In general, it is always possible to describe these images as a phasemodulated sinusoidal function. Examples of these methods are temporal phase-stepping interferometry and fringe projection profilometry with a spatial carrier. Usually the local phase can be approximated by a plane; that is, the fringe pattern is locally monochromatic. In this case the irradiance can be described locally by a sinusoidal signal of the form

$$
I(x)=b+m \cos (\omega x+\phi),
$$

where $b$ is the background, $m$ is the signal amplitude, and $\omega$ is the local frequency of the signal. The problem of phase detection consists in finding the value of the phase at the origin $\phi$. When the value of $\omega$ is known and is constant, we speak of synchronous phase detection. There are many well-known algorithms for

The authors are with the Departamento de Óptica, Facultad de Ciencias Fisicas, Universidad Complutense, Ciudad Universitaria s/n, 28040 Madrid, Spain. D. Crespo's e-mail address is daniel. crespo@indizen.com.

Received 26 August 2005; revised 14 November 2005; accepted 15 January 2006; posted 17 January 2006 (Doc. ID 64431).

0003-6935/06/174037-09\$15.00/0

C 2006 Optical Society of America synchronous phase detection and several general studies about the design techniques and properties of synchronous algorithms; in particular, one of the most powerful is the Fourier technique.,1,2

When the value of $\omega$ is not known or is not constant, then the problem is called asynchronous phase detection. This kind of problem arises in temporal phase shifting when the phase steps are not known a priori, or in spatial demodulation of fringe patterns with a carrier in which the local spatial frequency is not constant. For this kind of method, both the local frequency and the phase itself are obtained as the output. Normally what we get is the modulo $2 \pi$ version of the continuous phase: The wrapped phase is denoted by $W(\phi)$. Current asynchronous methods ${ }^{3-7}$ assume that the value of $\omega$ is locally constant and that the signal is sampled at $n$ points around the origin. They solve an algebraic system with $n \geq 4$ equations to solve for the unknowns $m, b, \omega$, and $\phi$. In the absence of noise, and for a signal with a constant local frequency, they yield the exact value of $\phi$.

Most phase detection methods, either synchronous or asynchronous, calculate the phase as the angle of the analytic signal associated with the fringe pattern of Eq. (1), which can be written as $A=C+i S$ and from which the wrapped phase is obtained as

$$
W(\phi)=\tan ^{-1}\left(\frac{S}{C}\right)
$$


$S$ and $C$ are obtained from the measured irradiances in the sampling points, and their expression depends on the particular method used. The magnitude of $A$ is given by

$$
m_{\phi}(\omega)=m m_{D}(\omega)=\sqrt{S^{2}(\omega)+C^{2}(\omega)},
$$

with this magnitude being composed of two terms, the amplitude of the fringe pattern $m$-coming from the experimental setup-and the response of the demodulation algorithm $m_{D}$, which will be a function of the local signal frequency $\omega$.

When problems associated with real signals as noise or nonlinearities are present, the effect on the signal is translated on the complex plane into a circle of uncertainty around the correct value of $A .^{8}$ The same level of noise is translated into a significant error in the detection of the phase when the value of $m_{\phi}(\omega)$ is small and into a small error when $m_{\phi}(\omega)$ is high. The signal amplitude $m$ is imposed by the experimental setup, illumination type, shadows, etc. and can always be optimized. On the other hand, $m_{D}(\omega)$ is determined by the particular algorithm. Therefore the algorithm response will be related to the signal-to-noise ratio (SNR) of the recovered phase and it will be an important characteristic of any asynchronous phase detection algorithm.

For example, the Carré technique ${ }^{3}$ uses four samples of the irradiance given by $I_{j}=b+m \cos [\phi$ $+(2 j-5) \omega]$ with $j=\{1,2,3,4\}$. After some algebra, the phase can be obtained as $\tan \phi=\left[3\left(I_{2}\right.\right.$ $\left.\left.-I_{3}\right)^{2}-\left(I_{1}-I_{4}\right)^{2}+2\left(I_{1}-I_{4}\right)\left(I_{2}-I_{3}\right)\right]^{1 / 2} /\left[\left(I_{2}+I_{3}\right)\right.$ $\left.-\left(I_{1}+I_{4}\right)\right]$. In this case it is easy to demonstrate that $m_{D}(\omega)=2 \cos 3 \omega-\cos \omega$. This function has a maximum for $\omega \approx 55^{\circ} /$ sample that coincides with the well-known result that the samples in the Carré method should be separated by $110^{\circ}$ to obtain optimum performance in front of additive noise.

With the same assumptions of harmonic behavior of the irradiance and local monochromaticity, there exist asynchronous techniques that use three ${ }^{5}$ (for signals without background), four, ${ }^{3}$ or five ${ }^{4,6,7}$ sampling points. As mentioned above, for a noiseless signal, all of these algorithms provide an exact value of the modulating phase regardless of the local signal frequency. But to check their performance in a widespectrum noisy signal it will be necessary to look at their respective algorithm response functions to estimate the recovered phase reliability as a function of the frequency. Figure 1 shows $m_{D}(\omega)$ for the Carré ${ }^{3}$ algorithm (solid curve) and the Servin and Cuevas ${ }^{5}$ and the Gomez-Pedrero et al. ${ }^{7}$ (dotted curve) algorithms. As can be seen, both algorithms have an optimum working point for a particular value of $\omega$, but their response becomes lower as the frequency changes, especially for low and high frequencies. In the case of the Carré method, there is also a low response zone for $\omega \approx 0.5 \pi \mathrm{rad} / \mathrm{sample}$.

In this work, we present a Fourier-based method to design asynchronous algorithms for harmonic and locally monochromatic signals. This technique allows

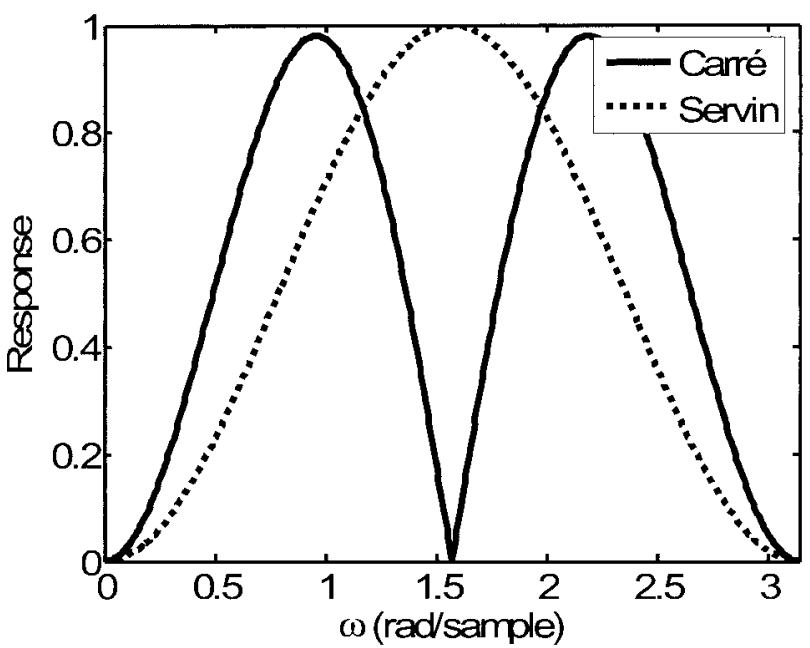

Fig. 1. Response as a function of frequency $\omega$ in radians per sample for the Carré ${ }^{3}$ algorithm (solid curve) and the Servin and Cuevas ${ }^{5}$ and the Gomez-Pedrero et al ${ }^{7}$ algorithms (dotted curve).

for the systematic development of asynchronous techniques with predefined response curves. The design criterion is to approximate any desired response function with a few sampling points while keeping the algebraic error within a known limit. The generated asynchronous methods are not algebraically exact, but they have better performance in terms of SNR than existing asynchronous methods for real noisy signals where the phase frequency has a large dynamic range.

Another interesting approach to the problem of signal asynchronous demodulation is the timefrequency analysis methods, namely, wavelet analysis, ${ }^{9,10}$ short-time Fourier transform, ${ }^{11,12}$ and Gabor filter bank. ${ }^{13}$ In these methods the local frequency at a given position is detected by means of correlation operations with a bank of band-limited spatially localized monochromatic signals (usually Gaussian shaped because this is the best choice in terms of time-frequency localization). These methods are designed in terms of the signal spectrum and they are computing time and memory resources intensive (for example, for a one-dimensional signal, a twodimensional transform should be calculated and analyzed); consequently they are not well suited for fast demodulation of two-dimensional fringe patterns. However, the big advantage of these time-frequency techniques is their capability to cope with nonsinusoidal signals. On the other hand, the Fourier technique we present permits the design of small convolution kernels-similar to the case for the Carré technique-with the desired response function, which is appropriate for fast asynchronous demodulation but that relies on the sinusoidal nature of Eq. (1).

This work is organized as follows. In Section 2 we present the theoretical foundations of the proposed method. In Section 3 we show the performance of one asynchronous algorithm designed with the new 
methodology with simulated as well as experimental data. Finally, in Section 4 we provide our conclusions.

\section{Proposed Method}

\section{A. Theoretical Foundations}

For simplicity we will assume that the background $b$ is filtered out; as we show in Subsection 2.C, this task can be easily accomplished within the framework of the proposed technique. Consequently, the periodic signal around the point of interest can be expressed as

$$
I(x)=m \cos (\omega x+\phi),
$$

where $m, \phi$, and $\omega$ are unknowns. We will make the assumption that this signal is sampled at equally spaced intervals, centered on the origin, with a sampling frequency $\omega_{s}$. If the signal is well sampled, there will be at least two samples for each signal period that in terms of the sampling frequency can be expressed by the well-known Nyquist condition $\omega_{s}>2 \omega$. We will define the normalized local frequency as the magnitude $\sigma=\omega / \omega_{s}$, which therefore can hold values between 0 and 0.5 for well-sampled signals.

The sampled signal corresponding to Eq. (4) can be represented by the following expression:

$$
\begin{aligned}
I_{s}(x) & =\sum_{n=-\infty}^{\infty} \delta\left(x-n p_{s}\right) I(x) \\
& =m \sum_{n=-\infty}^{\infty} \delta\left(x-n p_{s}\right) \cos (2 \pi n \sigma+\phi),
\end{aligned}
$$

where $p_{s}=2 \pi / \omega_{s}$ is the sampling period.

We will study the case of linear algorithms; we will consider demodulation algorithms whose quadrature components $C$ and $S$ are defined by two linear functions of the sampled values of the signal, that is,

$$
\begin{aligned}
C(\phi) & =\int \mathrm{d} x \sum_{n=-\infty}^{\infty} c(n) \delta\left(x-n p_{s}\right) I(x) \\
& =\sum_{n=-\infty}^{\infty} c(n) I\left(n p_{s}\right), \\
S(\phi) & =\int \mathrm{d} x \sum_{n=-\infty}^{\infty} s(n) \delta\left(x-n p_{s}\right) I(x) \\
& =\sum_{n=-\infty}^{\infty} s(n) I\left(n p_{s}\right) .
\end{aligned}
$$

These functions cannot be arbitrary; they must fulfill Eq. (2) for the phase computation.

In the case of a harmonic signal we can substitute Eq. (4) into Eqs. (6) and obtain

$$
C(\phi)=m \sum_{n=-\infty}^{\infty} c(n) \cos (2 \pi n \sigma+\phi),
$$

$$
S(\phi)=m \sum_{n=-\infty}^{\infty} s(n) \cos (2 \pi n \sigma+\phi) .
$$

From Eqs. (2) and (7) it follows that, for linear methods, the problem of measuring $\phi$ is reduced to the task of finding two sets of coefficients, $c(n)$ and $s(n)$, such that they satisfy the following expression:

$$
\begin{aligned}
& \sum_{n=-\infty}^{\infty} c(n) \cos (2 \pi n \sigma+\phi)=\cos (\phi), \\
& \sum_{n=-\infty}^{\infty} s(n) \cos (2 \pi n \sigma+\phi)=\sin (\phi) .
\end{aligned}
$$

Using the cosine sum formula, Eqs. (8) can be rewritten as

$$
\begin{gathered}
\cos (\phi) \sum_{n=-\infty}^{\infty} c(n) \cos (2 \pi n \sigma)-\sin (\phi) \sum_{n=-\infty}^{\infty} c(n) \sin (2 \pi n \sigma) \\
=\cos (\phi), \\
\cos (\phi) \sum_{n=-\infty}^{\infty} s(n) \cos (2 \pi n \sigma)-\sin (\phi) \sum_{n=-\infty}^{\infty} s(n) \sin (2 \pi n \sigma) \\
=\sin (\phi) .
\end{gathered}
$$

So that Eqs. (9) can be true for any value of $\phi$, the following conditions must be true:

$$
\begin{aligned}
& \sum_{n=-\infty}^{\infty} c(n) \sin (2 \pi n \sigma)=0, \\
& \sum_{n=-\infty}^{\infty} s(n) \cos (2 \pi n \sigma)=0 .
\end{aligned}
$$

And now, for Eqs. (10) to be true for any value of $\sigma$, it is necessary that

$$
\begin{aligned}
& c(n)=c(-n), \\
& s(n)=-s(-n) .
\end{aligned}
$$

This means that the sampling function $c(n)$ must be symmetrical around the origin and $s(n)$ must be antisymmetrical.

Assuming that Eqs. (11) are true, we can then rewrite Eqs. (7) as

$$
\begin{aligned}
& C(\phi)=\cos (\phi) m M_{C}(\sigma), \\
& S(\phi)=\sin (\phi) m M_{S}(\sigma),
\end{aligned}
$$

where we have defined the functions $M_{C}(\sigma)$ and $M_{S}(\sigma)$ as

$$
M_{C}(\sigma)=\sum_{n=-\infty}^{\infty} c(n) \cos (2 \pi n \sigma),
$$




$$
M_{S}(\sigma)=\sum_{n=-\infty}^{\infty} s(n) \sin (2 \pi n \sigma)
$$

$M_{C}(\sigma)$ is a symmetric and periodic function of $\sigma$ with period 1 , and $M_{S}(\sigma)$ is an antisymmetric and periodic function of $\sigma$ with the same period. Using Eqs. (11), Eqs. (13) can also be expressed as

$$
\begin{aligned}
& M_{C}(\sigma)=\sum_{n=-\infty}^{\infty} c(n) \exp (i 2 \pi n \sigma), \\
& M_{S}(\sigma)=i \sum_{n=-\infty}^{\infty} s(n) \exp (i 2 \pi n \sigma) .
\end{aligned}
$$

This equation shows that $c(n)$ are the coefficients of the Fourier series expansion of $M_{C}(\sigma)$ and $s(n)$ are the imaginary parts of the coefficients of the Fourier series expansion of $M_{S}(\sigma)$.

Now, using Eqs. (2) and (12) to obtain the estimated phase value $\phi_{e}$, we obtain

$$
\phi_{e}=\arctan \left[\frac{S(\phi)}{C(\phi)}\right]=\arctan \left[\frac{M_{S}(\sigma) \sin (\phi)}{M_{C}(\sigma) \cos (\phi)}\right],
$$

and the algorithm response as defined in Eq. (3) will be given by

$$
m_{D}(\sigma)=\sqrt{M_{S}(\sigma)^{2}+M_{C}(\sigma)^{2}}
$$

From Eqs. (13), under a first-order approximation, the algebraic error in the recovered phase will be limited by

$$
\varepsilon(\sigma)=\left|\phi_{e}-\phi\right| \approx \frac{1}{2}\left|\frac{M_{S}(\sigma)}{M_{C}(\sigma)}-1\right| .
$$

The previous discussion can be summarized in the following proposition: Given a pair of functions, $M_{C}(\sigma)$ real, symmetrical, and periodic in $\sigma$ with period 1 , and $M_{S}(\sigma)$ real, antisymmetrical, and periodic in $\sigma$ with period 1 , we can define an algorithm for asynchronous phase detection, which will be given by Eqs. (6) where $c(n)$ and $s(n)$ are given by the Fourier series coefficients of $M_{C}(\sigma)$ and $M_{S}(\sigma)$, as shown in Eqs. (14). The maximum algebraic error of this algorithm for any value of $\sigma$ will be given by Eq. (17), and the frequency response of the algorithm will be given by Eq. (16). In consequence, selecting the appropriate functions $M_{C}(\sigma)$ and $M_{S}(\sigma)$, we will have an algorithm with any desired frequency response curve.

he mathematical treatment shown in this subsection is similar to the one that has been used in previous works for Fourier description of synchronous phase detection with discrete sampling signals. ${ }^{2}$ This formalism has been used by other authors to design synchronous methods and study their properties. In our case, the novelty of the proposed technique is that we reinterpret this theory and use it to design asynchronous algorithms with desired response properties.
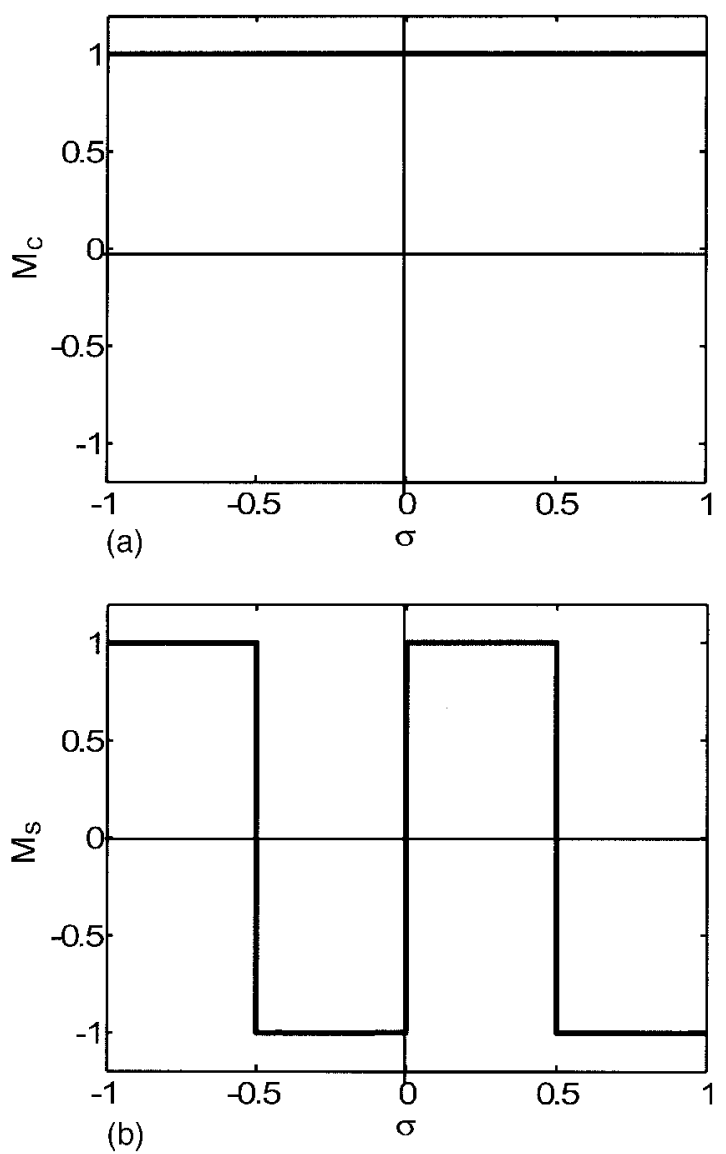

Fig. 2. Functions that define the optimum asynchronous algorithm for all values of $\sigma \in(0,0.5)$ : (a) symmetric $M_{C}(\sigma)$ and (b) antisymmetric $M_{S}(\sigma)$.

\section{B. Optimum Algorithm}

First, we show how this method can be used to design an optimum asynchronous algorithm for harmonic signals with no background. By optimum we mean that the algorithm has no algebraic error and has a constant algorithm response for any value of $\sigma$. This means that both $M_{C}(\sigma)$ and $M_{S}(\sigma)$ must be equal to 1 in the whole interval $(0,0.5)$ and satisfy the symmetry conditions explained in Subsection 2.A. Such a pair of functions is shown in Fig. 2, where $M_{C}(\sigma)$ is constant and $M_{S}(\sigma)$ is a square wave with period 1 . From Eqs. (6) and (15), it follows that the phase algorithm will be given by

$$
\tan \phi=\frac{\sum_{n=-\infty}^{\infty} s(n) I(n)}{\sum_{n=-\infty}^{\infty} c(n) I(n)},
$$

where $I(n)$ denotes the signal sampled at the $n$th point around the origin and, as we showed in Eqs. (14), $s(n)$ and $c(n)$ correspond to the terms in the Fourier series expansion of $M_{S}(\sigma)$ and $M_{C}(\sigma)$, respectively.

So, substituting the Fourier series terms of a square wave and a constant function, ${ }^{14}$ we obtain 
$c(n)=\delta(n)$ and $s(2 n+1)=2 / \pi(2 n+1), s(2 n)=0$; therefore the asynchronous algorithm defined by the two functions shown in Fig. 2 will be

$$
\tan \phi=\frac{\sum_{n=0}^{x} \frac{2}{\pi(2 n+1)}[I(2 n+1)-I(-2 n-1)]}{I(0)} .
$$

In a theoretical case, this is the optimum algorithm, with 0 phase error and maximum response for any signal frequency. But it is not useful in practice, since it is based on the assumptions that signal background is nonexistent and that infinite sampling points are contributing to each measurement. Also, the presence in the denominator of Eq. (19) of $I(0)$ implies that this method will be highly sensitive to noise, regardless of the number of sampling points.

\section{Background Suppression}

The previous discussion was based on the assumption that the signal background $b$ could be suppressed. Usually, this is a strong assumption, and an algorithm for phase detection should be able to work with signals with a local background. Introducing the background term into the left side of Eqs. (8) yields one extra term that must cancel out:

$$
\begin{gathered}
\sum_{n=-\infty}^{\infty} c(n)=0, \\
\sum_{n=-\infty}^{\infty} s(n)=0 .
\end{gathered}
$$

The second of these expressions is always true because $s(n)$ is antisymmetrical. The first line of Eqs. (20) implies one extra condition that must be satisfied by $M_{C}(\sigma)$, which is $M_{C}(0)=0$.

This means that any background-insensitive algorithm will not work properly for $\sigma=0$. However, for any value $\mu$ arbitrarily small, we can define an optimum algorithm for the range $\sigma \in(\mu, 0.5)$. The function $M_{C}(\sigma)$ would be, in this case, the one shown in Fig. 3, a symmetric periodic function that has a value of 1 for $\sigma \in(\mu, 0.5)$ and is 0 everywhere else. $M_{S}(\sigma)$ is the same as shown in Fig. 2(b) since the background suppression does not affect the antisymmetric kernel. In this case, using the Fourier series terms of a square wave with a duty cycle of $\mu / 2$ for $M_{C}(\sigma)$ (as shown in Fig. 4), we obtain $c(n)=\sin (2 n \pi \mu) / n \pi$, $c(0)=1-2 \mu$, and $s(n)$ is the same as before. ${ }^{14}$ Therefore the optimum asynchronous algorithm will be given by

$$
\begin{aligned}
& \tan \phi= \\
& \frac{\sum_{n=0}^{\infty} \frac{2}{\pi(2 n+1)}[I(2 n+1)-I(-2 n-1)]}{(1-2 \mu) I(0)-\sum_{n=1}^{\infty} \frac{\sin (2 n \pi \mu)}{n \pi}[I(n)+I(-n)]} .
\end{aligned}
$$

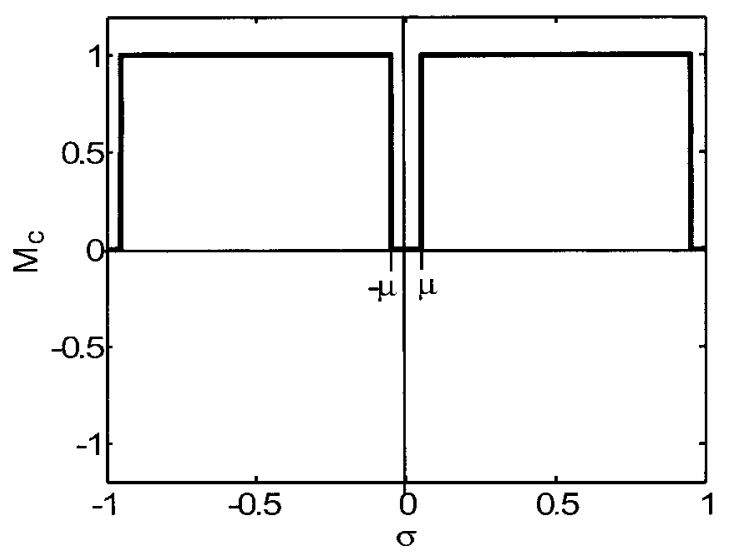

Fig. 3. Symmetric function $M_{C}(\sigma)$ that defines the optimum background-insensitive algorithm for all values of $\sigma \in(\varepsilon, 0.5)$. The antisymmetric function $M_{S}(\sigma)$ is the same as depicted in Fig. 2(b).

This algorithm is background insensitive and has uniform response and 0 algebraic error for any value of $\sigma \in(\mu, 0.5)$.

\section{Limited Number of Harmonics}

The algorithms described above are optimum, but they assume that there is an infinite number of harmonics contributing to $M_{C}(\sigma)$ and $M_{S}(\sigma)$; this means that there are infinite sampling points contributing to the measurement of the phase. Obvi-
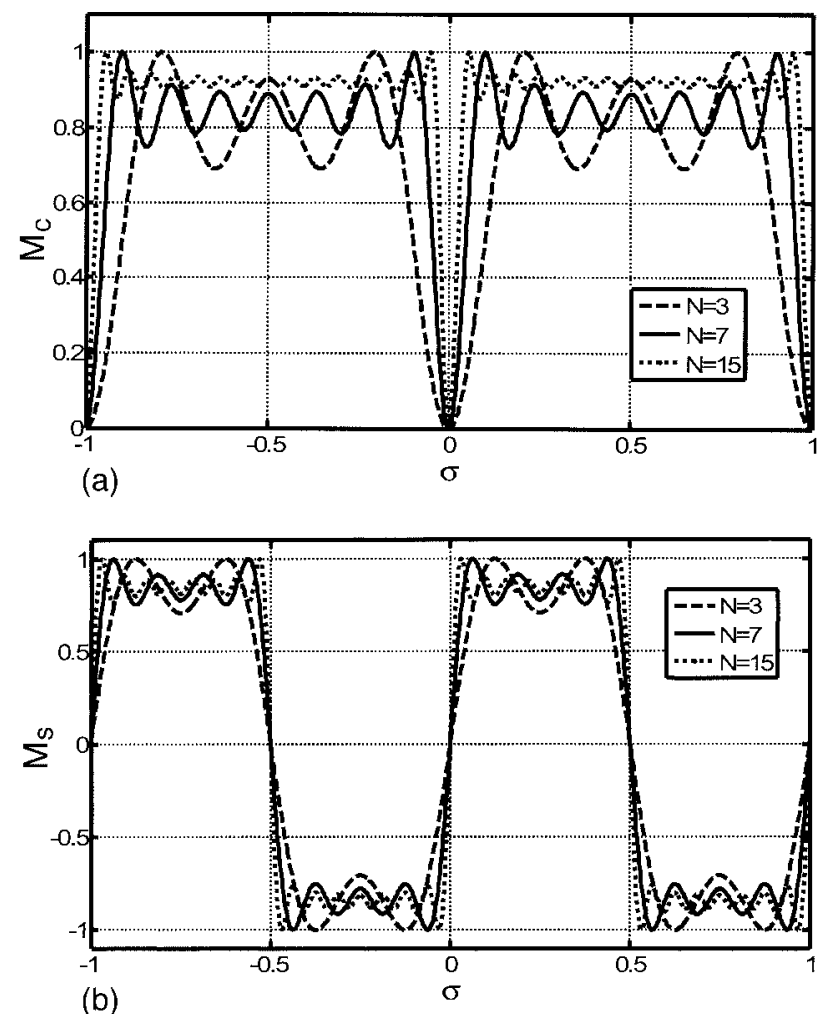

Fig. 4. Functions (a) $M_{C}(\sigma)$ and (b) $M_{S}(\sigma)$ that define the algorithm used in the experiment. These are the functions obtained from the optimum background-insensitive algorithm when $\mu=0.025$ and for different values of $N$. 


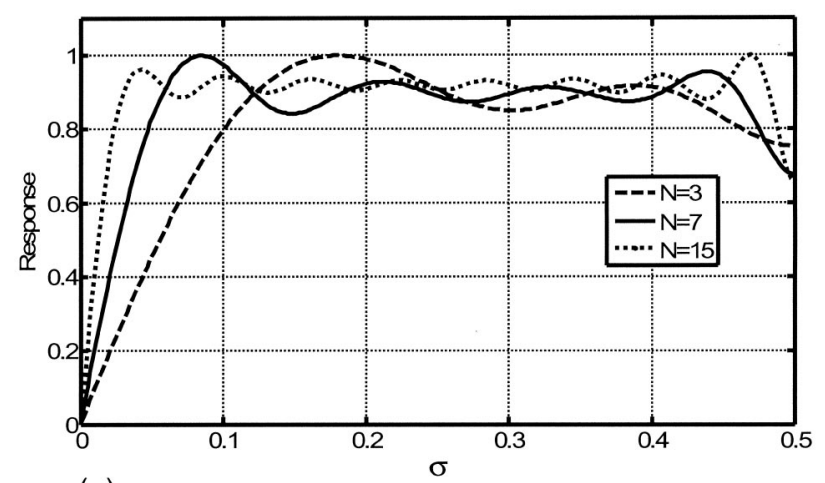

(a)

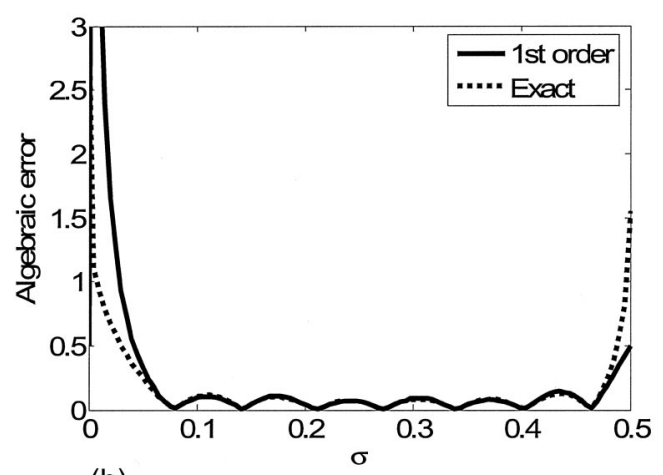

(b)

Fig. 5. (a) Response of the asynchronous algorithm defined by the functions shown in Fig. 4 for $N=3,7$, and 15. (b) Maximum algebraic error for the algorithm with $N=7$.

ously, this cannot be true in any practical situation; any practical algorithm can count only on a reduced number of samples around the point where the phase has to be measured. The main consequence of this is that the optimum sampling functions can be approximated only by their first $N$ terms in their Fourier series expansion $(|n| \leq N)$; this will correspond to $2 N+1$ sampling points. Figure 4 shows the approximation of the sampling functions $M_{S}(\sigma)$ and $M_{C}(\sigma)$ shown in Figs. 2(b) and 3 for a value of $\mu=0.025$ and using only the first $N$ terms of their Fourier series expansion. Plots for $N=3,7$, and 15 are shown that correspond to 7, 15, and 31 sampling points, respectively. In this case the algebraic error $\varepsilon$ is no longer 0 and the response is no longer constant for the whole range of $\sigma \in(\mu, 0.5)$. Figure 5(a) shows the response corresponding to the sampling functions of Fig. 4. It can be seen that the response for low frequencies improves as $N$ grows, tending toward the optimum response as the number of sampling points is increased. In Fig. 5(b) we show the maximum algebraic error for the case of $N$ $=7$ given by the approximated formula of Eq. (17) and the exact value of the phase error obtained numerically.

For a given limited number of harmonics there are no optimum algorithms; it is necessary therefore to find a compromise between algebraic error and a loss of algorithm response for a target range of signal frequencies. The methodology for algorithm design

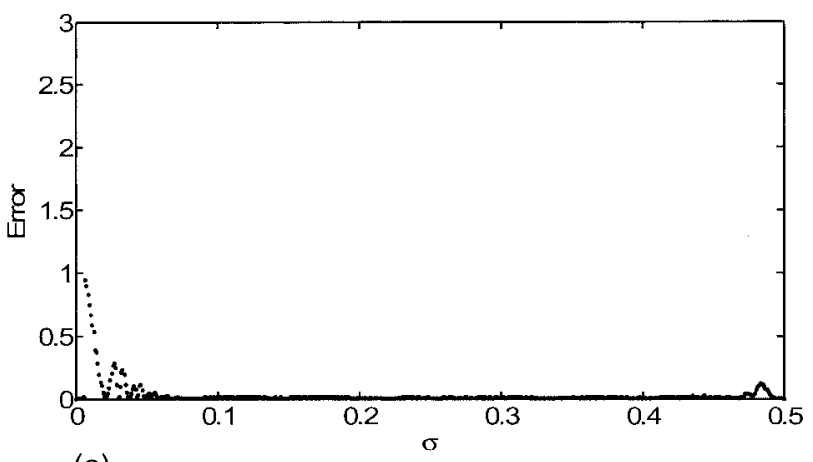

(a)



(b)

Fig. 6. Error in the recovered phase using (a) the new design algorithm and (b) the Gomez-Pedrero et al. ${ }^{7}$ algorithm.

presented in this work allows us to determine error and response values for any $\sigma$. With this information, we can build cost functions and make a systematic optimization process to find the best algorithm that meets a given set of design criteria. In some applications, it will be preferable to have small algebraic error with little concern for the SNR, whereas in other applications we may want to optimize only for a small range of values of $\sigma$. One example of cost function could be given by

$$
U_{N}[s(n), c(n)]=\int_{0}^{0.5}\left\{\left[1-m_{D}(\sigma)\right]^{2}+\beta \varepsilon^{2}(\sigma)\right\} \mathrm{d} \sigma,
$$

where $\beta$ is a coefficient that weights the importance of the algebraic error versus constant algorithm response. Substituting Eqs. (16) and (17) into Eq. (22), and substituting Eqs. (14) in the resulting expression, imposing the limit $|n| \leq N$, we obtain a function of $c(n)$ and $s(n)$ to be minimized. This minimization will yield the coefficients that define the best algorithm with $2 N+1$ sampling points, according to this particular cost function. Equation (22) represents a nonlinear minimization problem; thus a good seed point is necessary. In our approach one good possibility is to use the coefficients obtained from Eq. (21). 


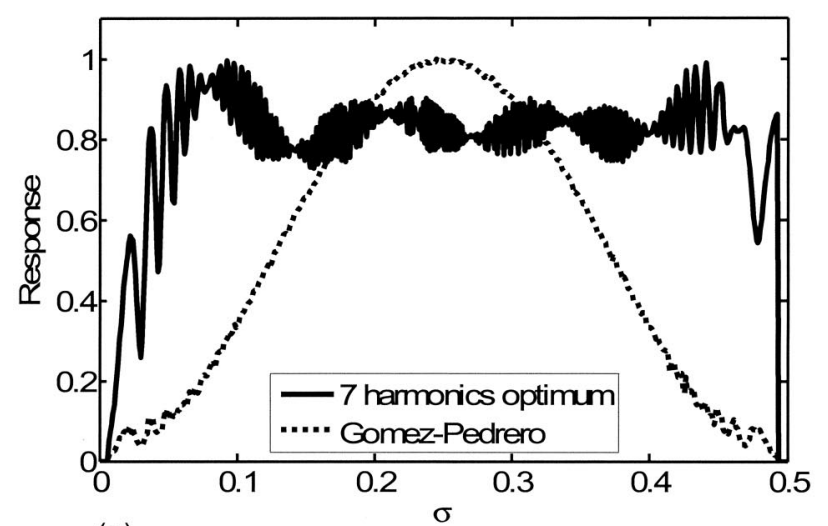

(a)



(b)

Fig. 7. (a) Comparison between the response obtained using the new design algorithm and the Gomez-Pedrero et al. algorithm. (b) Comparison between the experimental and the theoretical response of the algorithm.

\section{Results}

To illustrate the performance of the proposed method, we first applied the results obtained in this work to the demodulation of a numerically simulated fringe pattern. The fringe pattern consists of 128 rows, all of them with the same phase profile, but with independent additive phase noise for each row. Each line has 512 pixels and the phase at the $i$ th pixel is $\phi_{i}=i^{2} \pi / 1024$. In this experiment, the local signal for the $i$ th column is given by $I_{i}=100+60 \cos \left(\phi_{i}\right.$ $+r$ ), where $r$ is a normally distributed random function with a standard deviation of 0.1 . For each line, the value of the local signal frequency varies linearly from 0 to $\pi \mathrm{rad} /$ pixel from left to right. Since the sampling frequency is $2 \pi \mathrm{rad} /$ pixel (one sample per pixel), $\sigma$ ranges from 0 on the left side to 0.5 on the right side of each line.

We have demodulated this fringe pattern using the optimum algorithm with background suppression given by Eq. (21), with $\mu=0.025$, and using only the first \pm 7 harmonics $(N=7)$, which means that we use 15 sampling points for each measurement. The functions $M_{C}(\sigma)$ and $M_{S}(\sigma)$ associated with this algorithm are the ones shown in Fig. 4 with a solid curve; its theoretical response is shown in Fig. 5(a) with a solid curve, and the algebraic

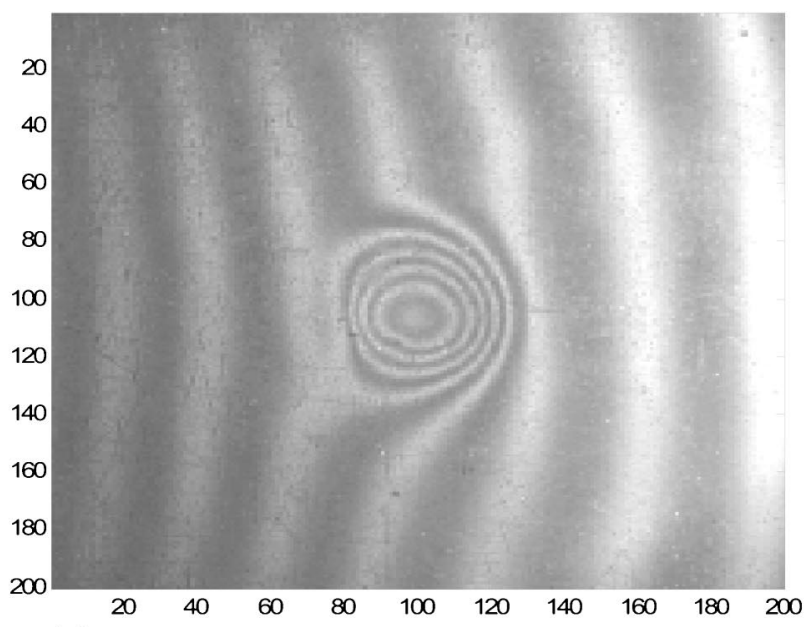

(a)

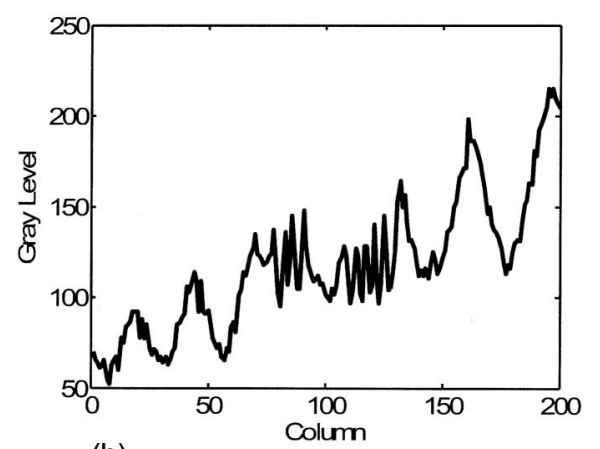

(b)

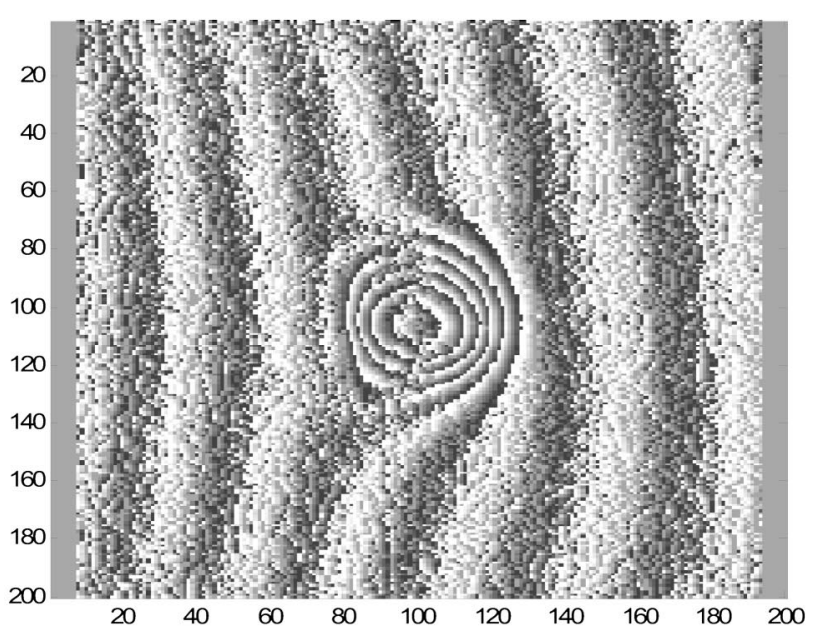

(c)

Fig. 8. (a) Experimental fringe pattern. (b) Profile of Fig. 7 along row 100, (c) phase recovered with the Gomez-Pedrero et al. algorithm.

maximum error is shown in Fig. 5(b). To test the performance of this response-oriented asynchronous method against a heuristically designed one, we have compared our results with those obtained with the five-step asynchronous algorithm by Gomez-Pedrero et al.,${ }^{7}$ which is insensitive to background, presents no algebraic error for all frequencies, and has the algorithm response shown with a dotted curve in Fig. 1. Figure 6 shows the errors 


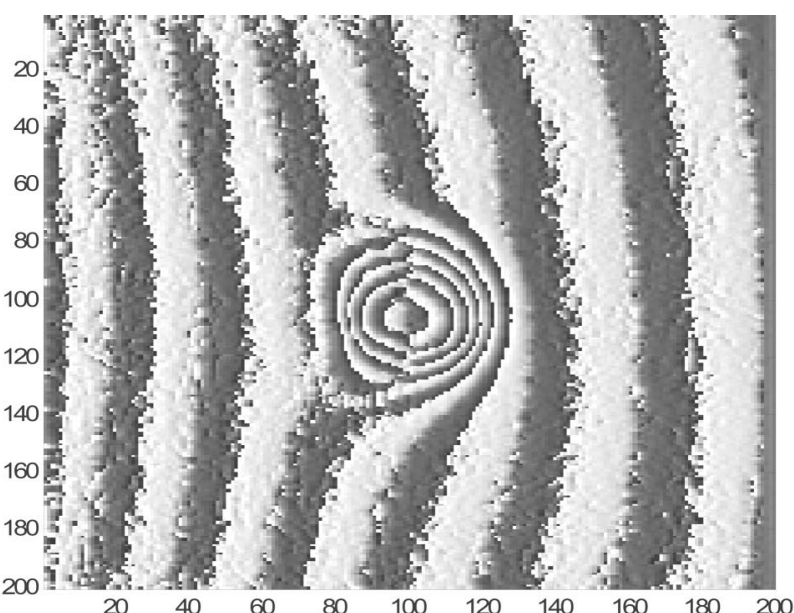

(a)

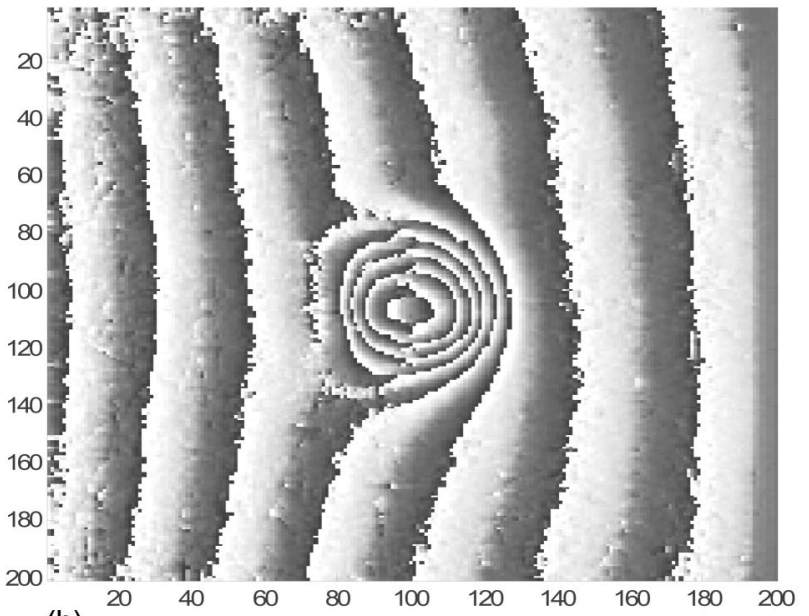

(b)

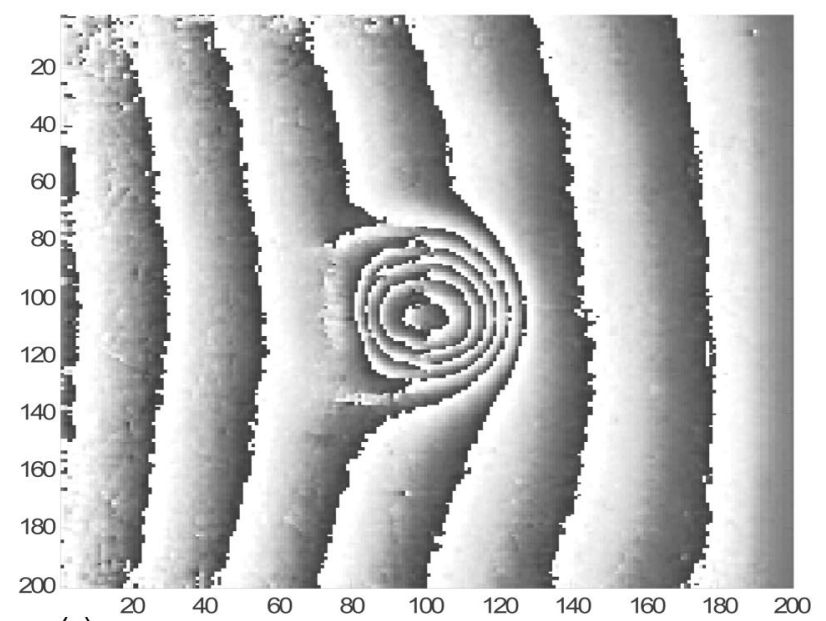

(c)

Fig. 9. Phase recovered from the image in Fig. 8(a) with the proposed algorithm for (a) $N=3$, (b) $N=7$, and (c) $N=15$.

made in phase detection with respect to theoretical values when demodulating with our algorithm and with that of Gomez-Pedrero et al. ${ }^{7}$ In each case, the graphs show the average error for the 128 lines of the synthetic fringe pattern. Our algorithm has a slightly higher error for the central frequencies, $\sigma \in(0.1,0.4)$, where both algorithms behave well and present little error. But for low frequencies, $\sigma<0.1$, and high frequencies, $\sigma>0.4$, our algorithm behaves much better and the errors in the recovered phase are much smaller. As mentioned, the Gomez-Pedrero et al. ${ }^{7}$ algorithm has no algebraic error, but in the presence of noise it yields poor results for low and high frequencies because the algorithm response for those frequencies is very low. In Fig. 7(a) we show the comparison between the response obtained with our algorithm and with that of Gomez-Pedrero et al. It can be clearly seen that the response with the new algorithm is higher for low and high frequencies; this accounts for the better behavior of the new algorithm in those frequency regions. In Fig. 7(b) we show the comparison of the theoretical and experimental response obtained with the new algorithm, and we see that the response obtained using Eq. (16) is reproduced in the experiment.

Finally, we illustrate the application of our algorithm to an experimental $200 \times 200$ pixels shadow moiré fringe pattern, shown in Fig. 8(a), with nonuniform background and noise. We have used the same background-suppressing algorithm described above with $\mu=0.025$ and for three different values of $N=3,7$, and 15 . As can be seen in the profile along row 100 of the image in Fig. 8(b), this fringe pattern has a nonuniform background and signal amplitude as well as a wide dynamic range of spatial frequencies that range from 7 fringes per field in the lowfrequency region to 50 fringes per field in the central closed fringe pattern. These spatial frequencies correspond to a normalized frequency variation of $0.035-0.25$, with this frequency range posing a challenge for a traditional asynchronous method. Figure 8(c) shows the demodulated phase obtained with the Gomez-Pedrero et al. method. Figures 9(a)-9(c) show the results obtained with our algorithms for $N=3,7$, and 15 , respectively. In all cases, the phase has been demodulated in the horizontal direction. As can be seen in Figs. 9(a)-9(c), the phase in the low-frequency regions of the image is recovered better for the algorithms with a higher value of $N$, reflecting the increasing response of the algorithms for low frequencies that was shown in Fig. 5(a). Even for the case with $N=3$, the phase recovered in the lowfrequency regions is better than the result obtained with a standard algorithm, shown in Fig. 8(c). This yields a noisy distribution due to its poor response for those frequencies. The phase recovery in the central areas with higher frequencies is correct with both methods because it corresponds to the case where both have high response values.

\section{Conclusions}

Historically, asynchronous phase detection algorithms have been designed heuristically to have no algebraic error in the detection of the phase, but with no special concern for frequency response. However, it is clear that a good asynchronous algorithm for real data must be conceived, not only to yield a correct 
value of the phase for any signal frequency, but also to yield high response. This is because low response of the algorithm translates into low SNR in the measured phase.

In this work we have presented a new methodology to design asynchronous phase detection algorithms, taking into account both algebraic error and response. The methodology can be summarized as follows: Given two real functions, $M_{C}(\sigma)$, symmetric and periodical with period 1 , and $M_{S}(\sigma)$, antisymmetrical and periodical with period 1 , this pair of functions defines an asynchronous algorithm given by Eqs. (14). The algebraic error and the response for any local frequency of the signal can be written in a close form and are given by Eqs. (17) and (16).

For harmonic signals with no background, we have shown how to design an algorithm that is optimum (no error and uniform response 1) for any value of the local signal frequency. We have then seen that there is an additional condition that must be satisfied for an algorithm to be insensitive to the local background, namely, that $M_{C}(0)=0$. We have shown that it is not possible to design a background-insensitive algorithm that is optimum for all frequencies, but it is possible to design one that is optimum for all frequencies above an arbitrary small value $\mu$.

We have discussed the effect of the limited number of sampling points [that are equivalent to a limited number of harmonics in the Fourier expansions of $M_{C}(\sigma)$ and $M_{S}(\sigma)$ ] in the design of these algorithms. We have seen that there are truly no optimum algorithms (in the sense of 0 error and uniform response for the whole frequency range) with a limited number of harmonics, but with the methodology presented in this work we can design the best possible asynchronous algorithms based on certain optimization criteria. This optimization can take into account the maximum tolerable error in the recovered phase and the minimum required response for any signal frequency.

Finally, we have shown some experimental results obtained with one of the algorithms designed with this method. We have seen that, in the presence of noise, this algorithm behaves better than previous existing algorithms for high and low signal frequen- cies and how this improvement is due to the higher algorithm response for those frequencies.

We acknowledge Spain's Ministerio de Ciencia y Tecnologia for providing economic support for this work under the auspices of project DP12002-02104.

\section{References}

1. K. Freischlad and C. L. Koliopoulos, "Fourier description of digital phase-measuring interferometry," J. Opt. Soc. Am. A 7, 542-551 (1990).

2. D. Malacara, M. Servin, and Z. Malcacara, Interferogram Analysis for Optical Testing (Marcel Dekker, 1998).

3. P. Carré, "Installation et utilisation du comparateur photoelectrique et interferentiel du Bureau International des Poids et Mesures," Metrologia 2, 13-23 (1966).

4. P. Hariharan, B. F. Oreb, and T. Eiju, "Digital phase-shifting interferometry: a simple error-compensating phase calculation algorithm," Appl. Opt. 26, 2504-2505 (1987).

5. M. Servin and F. J. Cuevas, "A novel technique for spatial phase-shifting interferometry," J. Mod. Opt. 42, 1853-1862 (1995).

6. K. G. Larkin, "Efficient nonlinear algorithm for envelope detection in white light interferometry," J. Opt. Soc. Am. A 13, 832-843 (1996).

7. J. A. Gomez-Pedrero, J. A. Quiroga, and M. Servin, "Asynchronous phase demodulation algorithm for temporal evaluation of fringe patterns with spatial carrier," J. Mod. Opt. 51, 97-109 (2004).

8. B. Ströbel, "Processing of interferometric phase maps as complex-valued phasor images," Appl. Opt. 35, 2192-2198 (1996).

9. M. Cherbuliez and P. Jacquot, "Phase computation through wavelet analysis: yesterday and nowadays," in Proceedings of Fringe'01: The Fourth International Workshop on Automatic Processing of Fringe Patterns, W. Ostes and W. Juptner, eds. (Elsevier, 2001), pp. 154-162.

10. J. Zhong and J. Weng, "Spatial carrier-fringe pattern analysis by means of wavelet transform: wavelet transform profilometry," Appl. Opt. 43, 4993-4998 (2004).

11. C. A. Sciammarella and T. Kim, "Determination of strains from fringe patterns using space-frequency representations," Opt. Eng. 42, 3182-3193 (2003).

12. Q. Kemao, "Windowed Fourier transform for fringe pattern analysis," Appl. Opt. 43, 2695-2702 (2004).

13. A. Asundi and W. Jun, "Strain contouring using Gabor filters: principle and algorithm," Opt. Eng. 41, 1400-1405 (2002).

14. J. W. Harris and H. Stocker, Handbook of Mathematics and Computational Science (Springer, 1998). 